JOURNAL OF THE

CHUNGCHEONG MATHEMATICAL SOCIETY

Volume 26, No. 3, August 2013

\title{
ANALYSIS OF THE VLASOV-POISSON EQUATION BY USING A VISCOSITY TERM
}

\author{
Boo-Yong Choi*, Sun-Bu Kang**, And Moon-Shik LeE***
}

\begin{abstract}
The well-known Vlasov-Poisson equation describes plasma physics as nonlinear first-order partial differential equations. Because of the nonlinear condition from the self consistency of the Vlasov-Poisson equation, many problems occur: the existence, the numerical solution, the convergence of the numerical solution, and so on. To solve the problems, a viscosity term (a second-order partial differential equation) is added. In a viscosity term, the VlasovPoisson equation changes into a parabolic equation like the FokkerPlanck equation. Therefore, the Schauder fixed point theorem and the classical results on parabolic equations can be used for analyzing the Vlasov-Poisson equation. The sequence and the convergence results are obtained from linearizing the Vlasove-Poisson equation by using a fixed point theorem and Gronwall's inequality. In numerical experiments, an implicit first-order scheme is used. The numerical results are tested using the changed viscosity terms.
\end{abstract}

\section{Introduction and preparation}

Plasma physics is a fascinating scientific domain where an applied mathematician and more specifically, a numerical analyst can find a variety of beautiful and difficult nonlinear problems of great practical interest. More details can be found elsewhere in $[1,3,5,6,7,9,11$, $12,13,14,16,17,18,19,23,20,21,22]$. However, because plasmas are hot, ionized gases that are composed of ions, electrons, and neutral atoms, it is difficult to experiment on neutral atoms for realtime and to understand the behavior of particles(ionized gases composed of ions,

Received March 13, 2013; Accepted July 08, 2013.

2010 Mathematics Subject Classification: 35D05.

Key words and phrases: the Vlasov-Poisson equation, a fixed point theory, the Schauder fixed point theorem, nonlinear problems, an implicit first-order scheme.

Correspondence should be addressed to Boo-Yong Choi, byc8734@hanmail.net.

*Supported by the Rearch Foundation at Korea Air Force Academy Project No. KAFA12-08. 
electrons, and neutral atoms). A very efficient tool for experiments is the computer simulation of the mathematical model of a plasma using a numerical method. The equation that describes the idealized plasma in a mathematical model is the Vlasov equation, which describes the electron distribution $u$,

$$
\frac{\partial u}{\partial t}+v \cdot \nabla_{x} u+F \cdot \nabla_{y} u=0, \quad x, y \in R^{3}, \quad t>0,
$$

as coupled to the Poisson equation

$$
\begin{aligned}
\Delta_{x} \Phi & =\rho=\int_{\Omega_{y}} u d y-1, \\
\nabla_{x} \Phi & =F
\end{aligned}
$$

where $\rho$ is the charge density. In an electrostatic case, $\nabla_{x}=\left(\partial / \partial x_{1}, \partial / \partial x_{2}\right.$, $\left.\partial / \partial x_{3}\right), \nabla_{y}=\left(\partial / \partial y_{1}, \partial / \partial y_{2}, \partial / \partial y_{3}\right), v=\left(v_{1}, v_{2}, v_{3}\right), F=\left(F_{1}, F_{2}, F_{3}\right)$ and $\Omega=\Omega_{x} \times \Omega_{y}$. To simplify and generalize the presentation of the method, the Vlasov equation is presented as follows:

$$
u_{t}+\zeta(u) \cdot \nabla u=0 .
$$

In this paper, let us assume that

$$
\zeta(u)=(v, F)=\left(v_{1}, v_{2}, v_{3}, F_{1}, F_{2}, F_{3}\right)
$$

and the particles are in a tokamak, where $\partial v_{i} / \partial x_{j}=0$ and $\partial F_{i} / \partial y_{j}=0$ for $i=1,2,3$ and $j=1,2,3$. More details can be found elsewhere in $[5,6]$.

The tokamak is characterized by azimuthal (rotational) symmetry, and the use of the plasma-borne electric current to generate the helical component of the magnetic field necessary for stable equilibrium. This can be contrasted to another toroidal magnetic confinement device, the stellarator, which has a discrete (e.g. fivefold) rotational symmetry, and in which all of the confining magnetic fields are produced by external coils with a negligible electric current flowing through the plasma. Hence the domain $\Omega$ is $S^{1} \times D^{5}$ in $R^{6}$, which is obtained by thickening the $S^{1}$ that is embedded in $R^{6}$. Its boundary is equal to $S^{1} \times S^{4}$. From (1.4) and $\zeta(u)$, the solution space has a compact support, where $\Omega_{x}=S^{1} \times D^{2}$, $\Omega_{y}=D^{3}$.

In the above equations, $u$ is the electron distribution function and $F$ is the electrostatic field. The charge density is known as $\rho$. Assuming a periodic plasma of period $L$, the functions $u$ and $F$ satisfy the periodic 
boundary conditions:

$$
\begin{aligned}
u\left(t, 0, x_{2}, x_{3}, y\right) & =u\left(t, L, x_{2}, x_{3}, y\right) \\
F\left(t, 0, x_{2}, x_{3}, y\right) & =F\left(t, L, x_{2}, x_{3}, y\right),
\end{aligned}
$$

where $\left(x_{2}, x_{3}, y\right) \in D^{5}$ for all $t \geq 0$. The periodic boundary condition is equivalent to

$$
\begin{gathered}
\int_{\Omega_{x}} \int_{\Omega_{y}} u d y d x=\left|\Omega_{x}\right| \\
\Leftrightarrow \int_{\partial \Omega_{x}} F \cdot n=0,
\end{gathered}
$$

where $n$ is a normal vector. To completely define the problem, a zeromean electrostatic field condition is added as follows

$$
\int_{\Omega_{x}} F=\overrightarrow{0}
$$

where $\overrightarrow{0}=(0,0,0)$ with an initial condition of $u(0, x, y)=u_{0}(x, y)$. More details can be found elsewhere in [9]. From the above conditions, and with $\Phi$ being defined up to an additive constant, the following equation is obtained

$$
\begin{aligned}
& \Delta_{x} \Phi=\rho=\int_{\Omega_{y}} u d y-1, \\
& \left.\Phi\right|_{\partial \Omega_{x}}=0 .
\end{aligned}
$$

By Poisson equation and Friedrichs' inequality,

$$
\begin{aligned}
\left\|\Delta_{x} \Phi\right\|_{L^{2}\left(\Omega_{x}\right)} & =\|\rho\|_{L^{2}\left(\Omega_{x}\right)} \leq C_{0}\|u\|_{L^{2}(\Omega)} \\
\|\nabla \Phi\|_{L^{2}\left(\Omega_{x}\right)} & \leq C_{1}\|u\|_{L^{2}(\Omega)},
\end{aligned}
$$

where $C_{0}, C_{1}$ are constants depending on $\Omega$. More details can be found elsewhere in $[4,10]$.

To show the existence of the solution of the Vlasov-Poisson system the second-order parabolic equation is used where $\|\zeta(u)\|_{L^{\infty}(\Omega)}$ is bounded. The Sobolev imbedding theorem is that if $m p>n$, then $W^{m, p}(\Omega) \subset L^{\infty}(\Omega)$, where $n$ is the dimension of $\Omega$ and $W$ is the Sobolev space. Since the dimension of $\Omega_{x}$ is 3 and $p$ is 2 , it is enough to make $m=2$. Then, $H^{2}\left(\Omega_{x}\right) \subset L^{\infty}\left(\Omega_{x}\right)$. Therefore, if $u \in H^{1}(\Omega)$, then $\rho \in H^{1}\left(\Omega_{x}\right)$. So, $\Phi \in H^{3}\left(\Omega_{x}\right)$ by Poisson's equation and $F_{i} \in H^{2}\left(\Omega_{x}\right)$ for $i=1,2,3$. $F_{i} \in L^{\infty}\left(\Omega_{x}\right)$ for $i=1,2,3$ are obtained. More details can be found elsewhere in [2]. 
In the following, let $\Omega$ denote an open subset of $R^{6}$, with boundary $\partial \Omega$ and $H^{k}(\Omega)$ as the Hilbert space for the norm

$$
\|u\|_{H^{k}(\Omega)}=\left(\sum_{|s| \leq k} \int_{\Omega}\left|D^{s} u(x)\right|^{2}\right) .
$$

The space $L^{p}\left(0, T ; H^{k}(\Omega)\right)$ consists of all functions $u$, such that for almost every $t$ in $(0, T), u(t)$ belongs to $H^{k}(\Omega) . \quad L^{p}\left(0, T ; H^{k}(\Omega)\right)$ is a normed space for the norm

$$
\|u\|_{L^{p}\left(0, T ; H^{k}(\Omega)\right)}=\left(\int_{0}^{T}\|u(t)\|_{H^{k}(\Omega)}^{p}\right)^{1 / p},
$$

where $p>1$ and $k$ is a positive integer. $L^{\infty}\left(0, T ; L^{2}(\Omega)\right)$ is a normed space for the norm

$$
\|u\|_{L^{\infty}\left(0, T ; L^{2}(\Omega)\right)}=e s s \sup _{0 \leq t \leq T}\|u(t)\|_{L^{2}(\Omega)} .
$$

Some other spaces that appear below can be defined analogously. More details can be found elsewhere in $[8,10,27]$.

The space $W(0, T)$ is introduced as follows:

$$
W(0, T)=\left\{w \in L^{2}\left(0, T ; H_{0}^{1}(\Omega)\right), \quad \frac{d w}{d t} \in L^{2}\left(0, T ; H^{1}(\Omega)^{\prime}\right)\right\},
$$

where $H^{1}(\Omega)^{\prime}$ is the dual space of $H_{0}^{1}(\Omega) . W(0, T)$ is a Hilbert space with the graph norm.

If $w \in W(0, T)$, then

$$
\rho_{w}=\int_{\Omega_{y}} w d y-1 \in H^{1}\left(\Omega_{x}\right) .
$$

By using the classical results of Poisson's equation and integration, the following equations are obtained

$$
\zeta(w) \in \prod^{6} L^{\infty}(\Omega)=\underbrace{L^{\infty}(\Omega) \times \ldots \times L^{\infty}(\Omega)}_{6}
$$

and

$$
\|\zeta(w)\|_{L^{\infty}(\Omega)} \leq M=\max \left\{\max _{\Omega_{y}}\|v\|_{\infty}, C_{0}\|w\|_{L^{2}(\Omega)}\right\},
$$

where $C_{0}$ is a constant depending on $\Omega$. 
For all $u, w \in W(0, T), \Phi_{u}$ and $\Phi_{w}$ can be obtained by Poisson's equation as follows: $\Delta_{x} \Phi_{u}=\rho_{u}$ and $\Delta_{x} \Phi_{w}=\rho_{w}$.

$$
\begin{aligned}
& \left\|\Delta_{x} \Phi_{u}-\Delta_{x} \Phi_{w}\right\|_{L^{2}\left(\Omega_{x}\right)}=\left\|\rho_{u}-\rho_{w}\right\|_{L^{2}\left(\Omega_{x}\right)} \\
& \left\|\nabla_{x} \Phi_{u}-\nabla_{x} \Phi_{w}\right\|_{L^{2}\left(\Omega_{x}\right)} \leq K\left\|\rho_{u}-\rho_{w}\right\|_{L^{2}\left(\Omega_{x}\right)}
\end{aligned}
$$

are obtained by Friedrichs' inequality, where $K$ is a constant. More details can be found elsewhere in $[4,10]$. By using the Corollary of the Sobolev imbedding theorem, the following equation is obtained

$$
\begin{aligned}
\left\|\nabla_{x} \Phi_{u}-\nabla_{x} \Phi_{w}\right\|_{L^{\infty}\left(\Omega_{x}\right)} & \leq K_{1}\left\|\nabla_{x} \Phi_{u}-\nabla_{x} \Phi_{w}\right\|_{L^{2}\left(\Omega_{x}\right)} \\
& \leq K_{2}\left\|\rho_{u}-\rho_{w}\right\|_{L^{2}\left(\Omega_{x}\right)} \leq K_{3}\|u-w\|_{L^{2}(\Omega)},
\end{aligned}
$$

where $K_{1}, K_{2}$ and $K_{3}$ are the constants.

Therefore, since $v=y,\|\zeta(u)-\zeta(w)\|_{L^{\infty}(\Omega)} \leq C_{1}\|u-w\|_{L^{2}(\Omega)}$ for all $u, w$ in $W(0, T)$ is obtained, where $C_{1}$ is a constant.

However, it is difficult to show the existence of the solution of (1.4), which is a nonlinear first-order partial differential equation. One of the methods for solving the problem is to add a second-order partial differential equation because the results (e.g. existence, uniqueness and regularity) of second-order partial differential equations are well known. Therefore, the (1.4) changes into

$$
u_{t}+\zeta(u) \cdot \nabla u-\nabla \cdot(\epsilon \nabla u)=0 .
$$

More details can be found elsewhere in [10, 27].

The paper is organized as follows: In Section 2, the existence and uniqueness of the proposed model Eq. (1.4) are shown. In Section 3, an iteration scheme, which converges to the solution of (1.4), is described. In section 4 , numerical experiments are shown.

\section{Existence and uniqueness results}

The standard notations will be used throughout this paper. More details can be found elsewhere in $[8,10,27]$.

Theorem 2.1. Let $u_{0} \in H_{0}^{1}(\Omega)$. Then, there is the unique weak solution $u$, such that $u \in C\left([0, T] ; L^{2}(\Omega)\right) \cap L^{2}\left(0, T ; H_{0}^{1}(\Omega)\right)$, with $d u / d t \in$ $L^{2}\left(0, T ; H^{1}(\Omega)^{\prime}\right)$, which satisfies

$$
\begin{array}{r}
u_{t}+\zeta(u) \cdot \nabla u-\nabla \cdot(\epsilon \nabla u)=0, \\
\left.u\right|_{\partial \Omega}=0, \\
u(0)=u_{0} .
\end{array}
$$


Proof. The proof consists of three parts:

1) the existence of a weak solution,

2) the regularity of the solution,

3) the uniqueness of the solution,

1) The existence of a weak solution. Unfortunately, the existence of the solution (1.4) does not always occur. To overcome this problem, the second-order parabolic equation theorem can be used by adding a secondorder term, $-\nabla \cdot(\epsilon \nabla u)$ to (1.4), as follows:

$$
u_{t}+\zeta(u) \cdot \nabla u-\nabla \cdot(\epsilon \nabla u)=0 .
$$

Since $\|\zeta(w)\|_{\infty}<M$, let $w \in W(0, T) \cap L^{\infty}\left(0, T ; L^{2}(\Omega)\right)$, such that

$$
\|w\|_{L^{\infty}\left(0, T ; L^{2}(\Omega)\right)} \leq\left\|u_{0}\right\|_{L^{2}(\Omega)} .
$$

From the linearization of (2.2) and the weak formulation, the following linear problem $[E w]$ is obtained: Find $u \in W(0, T)$, such that

$$
\begin{aligned}
\left(u_{t}, v\right)+(\zeta(w) \cdot \nabla u, v)+\epsilon(\nabla u, \nabla v) & =0, \quad \forall v \in H_{0}^{1}(\Omega) \text { a.e. in }[0, T] \\
u(0) & =u_{0} .
\end{aligned}
$$

Since $\zeta(w) \in \prod^{6} L^{\infty}(\Omega)$, there is a unique solution $U(w)$ of (2.3), which depends on $w$ and $\epsilon$. This follows from the classical results of the parabolic equation $[8,10,27]$. In fact, $U(w)$ is in the nonempty, convex, and weakly compact subset $W_{0}$ of $W(0, T)$ which is defined by

$$
\begin{array}{r}
W_{0}=\left\{w \in W(0, T):\|w\|_{L^{\infty}\left(0, T ; L^{2}(\Omega)\right)} \leq\left\|u_{0}\right\|_{L^{2}(\Omega)}, w(0)=u_{0},\right. \\
\left.\|w\|_{L^{2}\left(0, T ; H^{1}(\Omega)\right)}+\left\|w_{t}\right\|_{L^{2}\left(0, T ; H^{1}(\Omega)^{\prime}\right)} \leq C\left\|u_{0}\right\|_{L^{2}(\Omega)}\right\},
\end{array}
$$

where $C$ is a constant which only depends on $\Omega, T, \zeta(w)$ and $\epsilon$. Thus, $U$ is mapped from $W_{0}$ into $W_{0}$.

Since $W(0, T)$ is compactly imbedded into $L^{2}\left(0, T ; L^{2}(\Omega)\right), W_{0}$ is a closed, convex, bounded subset of $L^{2}\left(0, T ; L^{2}(\Omega)\right)$. In order to apply the Schauder fixed point theorem, it must be shown that $U(w)$ is a compact continuous mapping from $W_{0}$ into $W_{0}$. Let $\left\{w_{k}\right\}$ be a sequence in $W_{0}$ which converges weakly to some $w$ in $W_{0}$ and $u_{k}=U\left(w_{k}\right)$. The sequence $\left\{w_{k}\right\}$ contains a subsequence such that $u_{k} \rightarrow u$ weakly in $L^{2}\left(0, T ; H^{2}(\Omega)\right), d u_{k} / d t \rightarrow d u / d t$ weakly in $L^{2}\left(0, T ; H^{2}(\Omega)\right)$. More details can be found elsewhere in $[8,15,27]$. By passing to the limit in the 
relation

$$
\begin{aligned}
\left(\frac{d U\left(w_{k}\right)}{d t}(t), v\right)+\left(\zeta\left(w_{k}\right) \cdot \nabla U\left(w_{k}\right), v\right)+\epsilon\left(\nabla U\left(w_{k}\right), \nabla v\right) & =0 \\
U\left(w_{k}\right)(0) & =u_{0}
\end{aligned}
$$

$U\left(w_{k}\right) \rightarrow U(w)$ and $u=U(w)$ are obtained. Due to the uniqueness of the solution of $[E w]$, the whole sequence $u_{k}=U\left(w_{k}\right)$ converges weakly in $W(0, T)$ to $u=U(w)$. Hence, the mapping of $U$ is weakly continuous from $W_{0}$ into $W_{0}$. This, in turn, shows that the mapping of $U$ is compact. A similar argument shows that $U$ is continuously mapped. More details can be found elsewhere in $[8,15,27]$. By applying the Schauder fixed point theorem, $u$ exists such that $u=U(u)$, which consequently solves (2.3).

2) The regularity of the solution. Using the general theory of parabolic equations and the bootstrap argument, $u$ is a strong solution of $(2.3)$ and $u \in C^{\infty}((0, T) \times \Omega)$. More details can be found elsewhere in $[8,10,27]$.

3) The uniqueness of the solution. Let $u$ and $w$ be the two solutions for (2.2). For almost every $t$ in $[0, T]$,

$$
\begin{array}{r}
u_{t}+\zeta(u) \cdot \nabla u-\nabla \cdot(\epsilon \nabla u)=0, \\
w_{t}+\zeta(w) \cdot \nabla w-\nabla \cdot(\epsilon \nabla w)=0, \\
\text { and } u(0)=w(0)=u_{0} .
\end{array}
$$

By subtracting, multiplying and integrating the parts,

$$
\begin{aligned}
& \left((u-w)_{t}, v\right)+(\zeta(u) \cdot \nabla u-\zeta(w) \cdot \nabla w, v)+\epsilon(\nabla u-\nabla w, \nabla v)=0 \\
& \left((u-w)_{t}, v\right)+\epsilon(\nabla u-\nabla w, \nabla v) \\
& =-((\zeta(u)-\zeta(w)) \nabla u, v)-(\zeta(w)(\nabla u-\nabla w), v) .
\end{aligned}
$$

By taking $v=u-w$,

$$
\begin{aligned}
& \frac{1}{2} \frac{d}{d t}\|u-w\|_{L^{2}(\Omega)}^{2}+\epsilon\|\nabla u-\nabla w\|_{L^{2}(\Omega)}^{2} \\
& \leq\|\zeta(u)-\zeta(w)\|_{L^{\infty}(\Omega)}\|\nabla u\|_{L^{2}(\Omega)}\|u-w\|_{L^{2}(\Omega)} \\
& \quad+\|\zeta(w)\|_{L^{\infty}(\Omega)}\|\nabla u-\nabla w\|_{L^{2}(\Omega)}\|u-w\|_{L^{2}(\Omega)} .
\end{aligned}
$$

Moreover $\|\zeta(u)-\zeta(w)\|_{L^{\infty}(\Omega)} \leq C_{1}\|u-w\|_{L^{2}(\Omega)}$ and $\|\zeta(w)\|_{L^{\infty}(\Omega)} \leq M$.

By combining these inequalities and using Schwarz's inequality, (2.4) 
changes into

$$
\begin{aligned}
& \frac{1}{2} \frac{d}{d t}\|u-w\|_{L^{2}(\Omega)}^{2}+\epsilon\|\nabla u-\nabla w\|_{L^{2}(\Omega)}^{2} \\
& \leq C_{1}\|\nabla u\|_{L^{2}(\Omega)}\|u-w\|_{L^{2}(\Omega)}^{2} \\
& \quad+\epsilon_{1} M\|\nabla u-\nabla w\|_{L^{2}(\Omega)}^{2}+\frac{4 M}{\epsilon_{1}}\|u-w\|_{L^{2}(\Omega)}^{2} . \\
& \frac{1}{2} \frac{d}{d t}\|u-w\|_{L^{2}(\Omega)}^{2}+\left(\epsilon-\epsilon_{1} M\right)\|\nabla u-\nabla w\|_{L^{2}(\Omega)}^{2} \\
& \leq\left(C_{1}\|\nabla u\|_{L^{2}(\Omega)}+\frac{4 M}{\epsilon_{1}}\right)\|u-w\|_{L^{2}(\Omega)}^{2} .
\end{aligned}
$$

Assuming $0 \leq \epsilon-\epsilon_{1} M,(2.5)$ is changed into

$$
\frac{1}{2} \frac{d}{d t}\|u-w\|_{L^{2}(\Omega)}^{2} \leq C\|u-w\|_{L^{2}(\Omega)}^{2},
$$

where $C=\left(C_{1}\|\nabla u\|_{L^{2}(\Omega)}+4 M / \epsilon_{1}\right)$ is bounded by $u \in L^{2}\left(0, T ; H_{0}^{1}(\Omega)\right)$.

Since $u(0)=w(0)=u_{0},(2.6)$ and Gronwall's inequality yield $u(t, x)=$ $w(t, x)$ on $[0, T] \times \Omega$, which establishes the uniqueness.

\section{Convergent iterative scheme}

Discretised difference schemes will be used for the numerical experiments.

Theorem 3.1. Let $u_{0} \in H_{0}^{1}(\Omega)$. The sequence $\left\{u^{n}\right\}$ is defined by

$$
\begin{array}{r}
u_{t}^{n+1}+\zeta\left(u^{n}\right) \cdot \nabla u^{n+1}-\nabla \cdot\left(\epsilon \nabla u^{n+1}\right)=0, \\
\left.u^{n+1}\right|_{\partial \Omega}=0, \\
u^{n+1}(0)=u_{0},
\end{array}
$$

which converges in $C\left([0, T] ; L^{2}(\Omega)\right)$ to the strong solution of (2.2).

Proof. From (2.3), and assuming $u^{n} \in H_{0}^{1}(\Omega)$, the linear problem $E_{u^{n}}$ has a unique solution $u^{n+1}$. It is clear that $u^{n+1} \in H_{0}^{1}(\Omega)$ by Theorem 2.1 and the classical results of the parabolic equations. 
By using (2.4) in Theorem 2.1,

$$
\begin{aligned}
& \frac{1}{2} \frac{d}{d t}\left\|u-u^{n+1}\right\|_{L^{2}(\Omega)}^{2}+\epsilon\left\|\nabla u-\nabla u^{n+1}\right\|_{L^{2}(\Omega)}^{2} \\
& \leq\left\|\zeta(u)-\zeta\left(u^{n}\right)\right\|_{L_{0}^{\infty}(\Omega)}\|\nabla u\|_{L^{2}(\Omega)}\left\|u-u^{n+1}\right\|_{L^{2}(\Omega)} \\
& \quad+\left\|\zeta\left(u^{n}\right)\right\|_{L_{0}^{\infty}(\Omega)}\left\|\nabla u-\nabla u^{n+1}\right\|_{L^{2}(\Omega)}\left\|u-u^{n+1}\right\|_{L^{2}(\Omega)} .
\end{aligned}
$$

By using Schwarz's inequality, Poincare's inequality, $\epsilon$ and conditions of $\zeta$,

$$
\frac{1}{2} \frac{d}{d t}\left\|u-u^{n+1}\right\|_{L^{2}(\Omega)}^{2} \leq\|\nabla u\|_{L^{2}(\Omega)}^{2}\left\|u-u^{n}\right\|_{L^{2}(\Omega)}^{2} .
$$

Moreover

$$
\left\|u-u^{0}\right\|_{L^{2}(\Omega)}^{2} \leq C^{*} \forall t \in[0, T],
$$

where $C^{*}$ is a constant which only depends on $\zeta, \epsilon$ and $u_{0}$. Then, Gronwall's inequality yields, for any $t \in[0, T]$,

$$
\left\|u-u^{1}\right\|_{L^{2}(\Omega)}^{2} \leq C^{*}\left(\int_{0}^{T}\|\nabla u(s)\|_{L^{2}(\Omega)}^{2} d s\right),
$$

and, by iteration,

$$
\left\|u-u^{n+1}\right\|_{L^{2}(\Omega)}^{2} \leq \frac{C^{*}}{(n+1) !}\left(\int_{0}^{T}\|\nabla u(s)\|_{L^{2}(\Omega)}^{2} d s\right)^{n+1} .
$$

The sequence $\left\{u^{n}\right\}$ converges in $C\left([0, T] ; L^{2}(\Omega)\right)$ to the strong solution of $(2.2)$.

\section{Numerical experiments}

In order to simplify the presentation of the method for the numerical experiments, the 1D electrostatic case is presented as follows:

$$
\begin{aligned}
u_{t}+v \partial_{x} u & +F \partial_{y} u=0, \\
\Delta_{x} \Phi=\rho & =\int_{\Omega_{y}} u d y-1, \\
\left.\Phi\right|_{\partial \Omega_{x}} & =0 .
\end{aligned}
$$

By adding a second-order term and by using a standard finite differential method (an implicit first-order scheme), the 1D electrostatic case 
changes into

$$
\begin{aligned}
& \frac{u_{i, j}^{n+1}-u_{i, j}^{n}}{\triangle t}+v_{j}^{n} \frac{u_{i+1, j}^{n+1}-u_{i-1, j}^{n+1}}{2 \triangle x}+F_{i}^{n} \frac{u_{i, j+1}^{n+1}-u_{i, j-1}^{n+1}}{2 \triangle y} \\
& \quad-\epsilon\left(\frac{u_{i+1, j}^{n+1}-2 u_{i, j}^{n+1}+u_{i-1, j}^{n+1}}{(\triangle x)^{2}}+\frac{u_{i, j+1}^{n+1}-2 u_{i, j}^{n+1}+u_{i, j-1}^{n+1}}{(\triangle y)^{2}}\right)=0, \\
& v_{j}^{n}=y_{j} .
\end{aligned}
$$

The discrete problem can now be written

$$
\frac{u^{n+1}-u^{n}}{\triangle t}+A_{h}\left(\epsilon, u^{n}\right) u^{n+1}=0,
$$

where the matrix $A_{h}$ is positively defined. By classical arguments, $I+$ $\triangle t A_{h}\left(\epsilon, u^{n}\right)$ is invertible. More details can be found elsewhere in $[4,10$, $23]$.

It is hard to confirm the superiority of the methods. One verifiable means for confirmation is Landau damping. Landau damping occurs when the energy exchanges between a wave with phase velocity $V p h$ and particles in the plasma with velocity are approximately equal to $V p h$, which can interact strongly with the wave. Those particles that have velocities slightly less than $V p h$ will be accelerated by the wave electric field in order to move with the wave phase velocity, while those particles with velocities slightly greater than $V p h$ will be decelerated by the wave electric field, losing energy to the wave. In a collisionless plasma, the particle velocities are often taken to be approximately a Maxwellian distribution function. If the slope of the function is negative, the number of particles with velocities slightly less than the wave phase velocity is larger than the number of particles with velocities slightly greater. Hence, there are more particles that gain energy from the wave than lose energy to the wave, which leads to wave damping. If, however, the slope of the function is positive, the number of particles with velocities slightly less than the wave phase velocity is smaller than the number of particles that have velocities that are slightly greater. Hence, there are more particles that lose energy to the wave than gain energy from the wave, which leads to an increase in the wave energy.

To confirm this method, Finite Difference Method is used, where the initial data are given by

$$
u_{0}(x, y)=\frac{1}{\sqrt{2 \pi}} e^{-y^{2} / 2}(1+\alpha \cos k x),
$$


where $k=0.5,-5 \leq y \leq 5$, and $0 \leq x \leq 4 \pi$. The linear Landau damping theory is valid as long as $t<\alpha^{1 / 2}$ : For longer times, the problem is inherently nonlinear. Here, the Landau theory can not be applied because nonlinear effects are too important, despite the fact that this test has been studied numerically by many authors. The electric energy $\log \sum|F|$ first decays linearly and then periodically oscillates. More details can be found elsewhere in $[11,12,13,14,16,17,18,19,23$, 22].

In this case, $\alpha=0.01, \triangle t=0.01$, and $T=$ a number of iteration $\times \triangle t$. A number of cells $\left(N_{x}=32\right.$ in the $x$-direction and $N_{y}=32$ in the $y$-direction) are used.
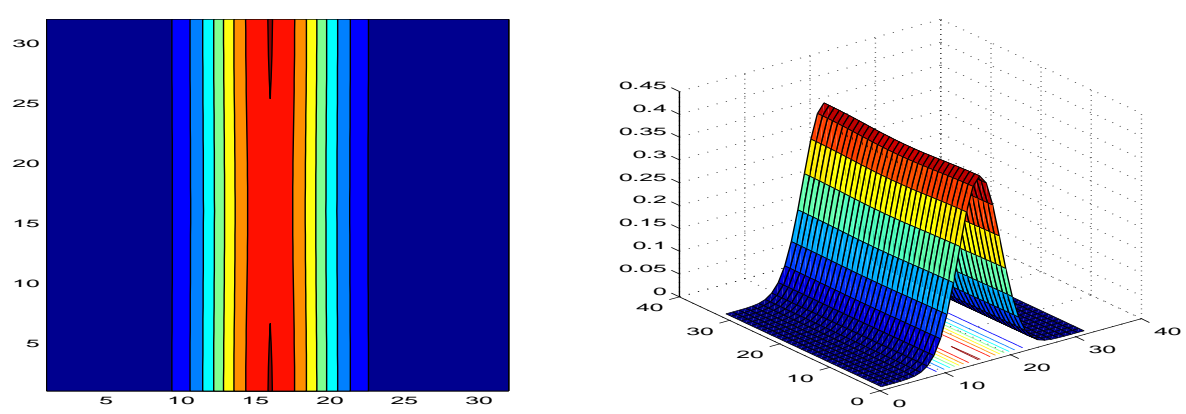

Figure 1.Initial condition $\alpha=0.01$

Figure 1 shows the initial function $u_{0}$ on $\alpha=0.01$. In Figure 1, the left image is a contour image and the right image is a $3-\mathrm{D}$ plot image.
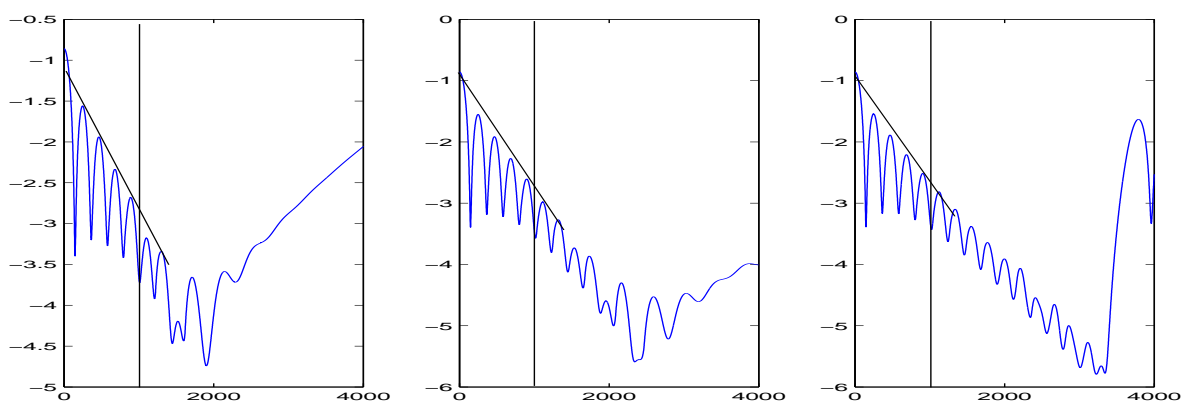

Figure 2. $\epsilon=0.01,0.005,0$

Figure 2 shows the electric energy $\log \sum|F|$ for $\epsilon(\epsilon=0.01,0.005,0)$. More details can be found elsewhere in [7, 11].

In this case, $\alpha=0.5, \triangle t=0.01$, and $T=$ a number of iteration $\times \triangle t$. A number of cells $\left(N_{x}=32\right.$ in the $x$-direction and $N_{y}=32$ in the $y$-direction) are used. 

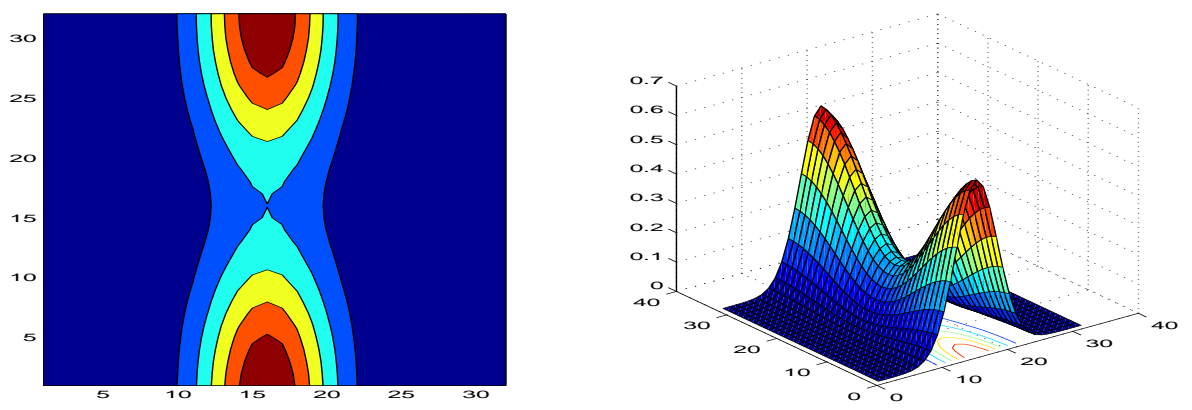

Figure 3. Initial condition $\alpha=0.5$

Figure 3 shows the initial function $u_{0}$ on $\alpha=0.5$. In Figure 3 , the left image is a contour image and the right image is a $3-\mathrm{D}$ plot image.
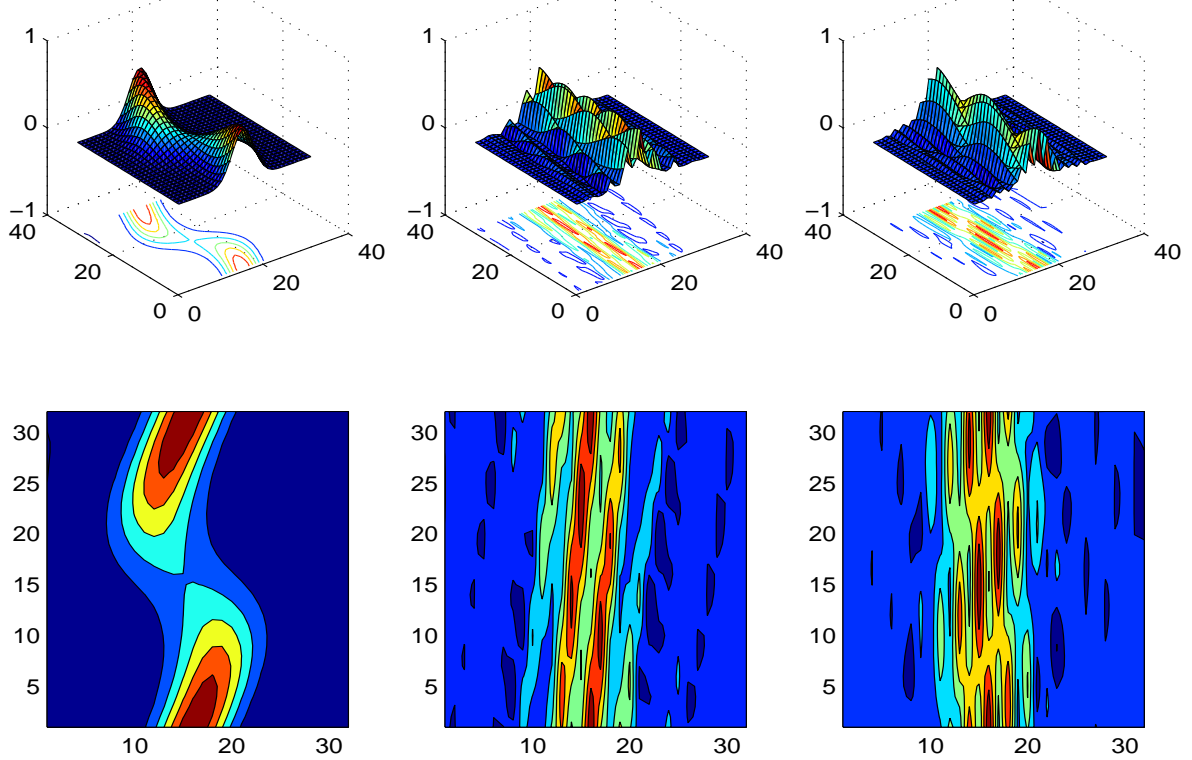

Figure 4. $T=1,10,20$

In Figure 4, the upper images are 3-D plot images and the lower images are contour images for each time $(T=1,10,20)$ on $\epsilon=0$. 

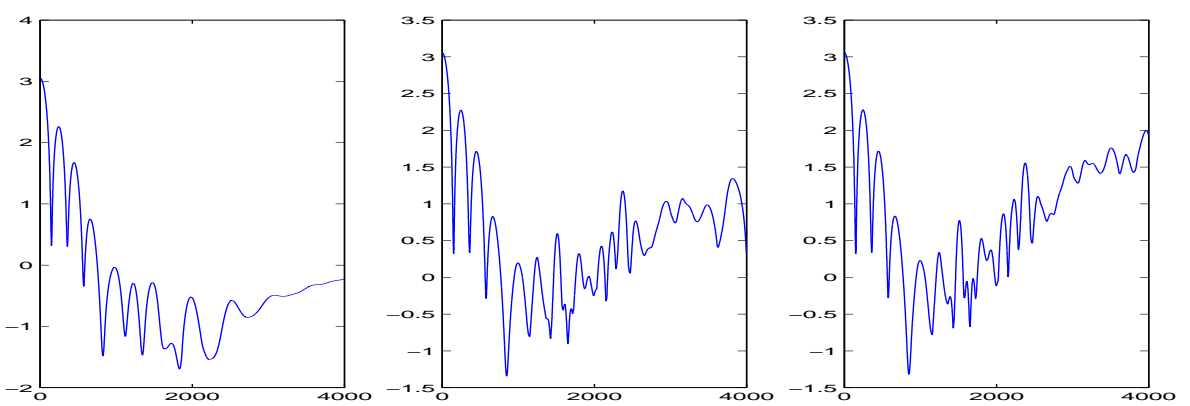

Figure 5. $\epsilon=0.01,0.001,0$

Figure 5 shows the electric energy $\log \sum|F|$ of results on $\alpha=0.5$ for $\epsilon$ $(\epsilon=0.01,0.001,0)$.

In this case, the initial data

$$
u_{0}(x, y)=\frac{y^{2}}{\sqrt{2 \pi}} e^{-y^{2} / 2}(1+\alpha \cos k x)
$$

are given, where $\triangle t=0.01$ and $T=$ a number of iteration $\times \triangle t$. A number of cells ( $N_{x}=32$ in the $x$-direction and $N_{y}=32$ in the $y$-direction) are used.

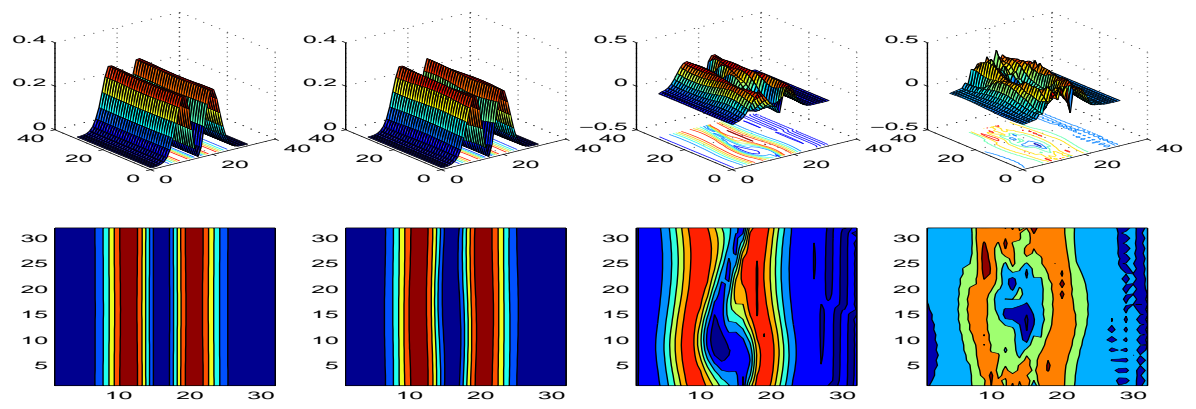

Figure 6. $T=0,10,20,30$

Figure 6 shows the results of condition $\alpha=0.01$. In Figure 6 , the upper images are $3-\mathrm{D}$ plot images and the lower images are contour images for each time $(T=0,10,20,30)$ on $\epsilon=0$. 

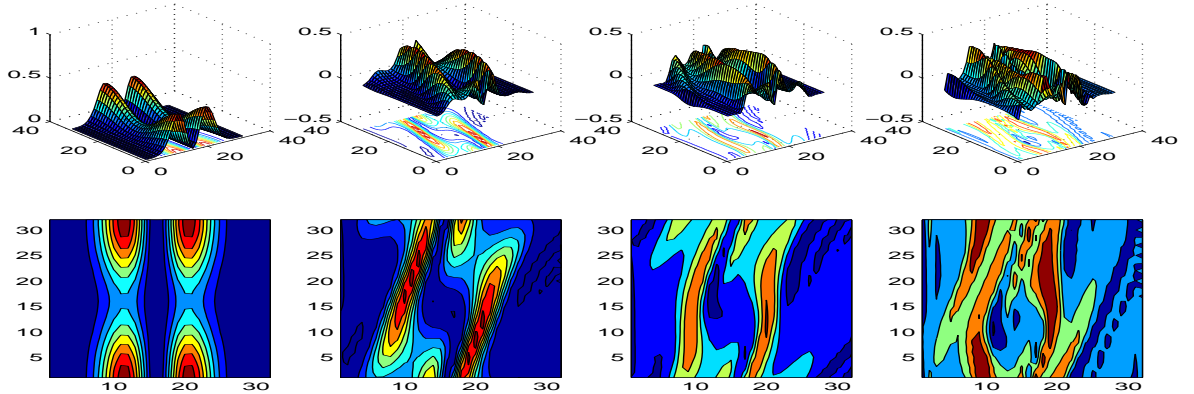

Figure 7. $T=0,3,4,6$

Figure 7 shows the results of condition $\alpha=0.5$. In Figure 7, the upper images are $3-\mathrm{D}$ plot images and the lower images are contour images for each time $(T=0,3,4,6)$ on $\epsilon=0$.

\section{Conclusion}

In this paper, the nonlinear first-order partial differential equation containing self consistence condition (the Vlasov-Poisson equation) was studied with a viscosity term. By using the viscosity term, this equation changes into a parabolic equation. Therefore, the well-known classical results of parabolic equations can be used. Our results have the viscosity effects. However, using small viscosity terms or without the viscosity term $(\epsilon=0)$ in numerical experiments, the results of proposed schemes are similar to the numerical results of the Vlasov-Poisson equation. Moreover, we analyzed the tokamak structure and obtained the confidential results of the long-time numerical solution, using the implicit scheme.

\section{References}

[1] A. Arakawa, Computational design for long-term numerical integration of the equation of fluid motion: Two-dimensional incompressible flow, Part 1, J. Comput. Phys. 1 (1966), no. 1, 119-143; Reprinted in J. Comput. Phys. 135 (1997), 103-114.

[2] R. Adams, Sobolev spaces, Academic Press, 1975.

[3] C. Bardos and P. Degond, Global existence for the Vlasov-Poisson equation in 3 space variables with small initial data, Ann. Inst. Henri Poincare 2 (1985), no. $2,101-118$.

[4] D. Braess, Finite elements, Cambrige, 2001.

[5] P. M. Bellan and B. L. Shader, Fundamentals of plasma physics, Cambrige, 2006. 
[6] J. Batt, Global symmetric solutions of the initial value problem of stellar dynamics, J. Differen. Equat. 25 (1977), 342-364.

[7] C. Z. Cheng and G. Knorr, The integration of the Vlasov equation in configuration space, J. Comput. Phys. 22 (1976), 330-351.

[8] F. Catte, P. L. Lions, J. M. Morel and T. Coll, Image selective smoothing and edge detection by nonlinear diffusion, SIAM. J. Numer. Anal., 29 (1) (1992), pp.182-193.

[9] G. H. Cottet and P. A. Raviart, Particle methods for the one-dimensional Vlasov-Poisson equations, SIAM. J. Numer. Anal., 21, (1) (1984), pp.52-76.

[10] L. C. Evans, Partial Differenctial Equations. Graduate Studies in Mathematics vol 19. American Mathematical Society Providence, Rhode Island., 1998.

[11] E. Fijakow, A numerical solution to the Vlasov equation, Comput. Phys. Comm. 116 (1999), 319-328.

[12] F. Filbet and E. Sonnendrucker, Comparison of eulerian Vlasov solvers, Com. Phys. Comm. 150 (2003), 247-266.

[13] F. Filbet, E. Sonnendrucker and P. Bertrand, Conservative numerical schemes for the Vlasov equation, J. Comput. Phys. 172 (2001), 166-187.

[14] R. R. Gagne and M. M. Shoucri, A splitting scheme for the numerical solution of a one-dimensional Vlasov equation, J. Comput. Phys. 24 (1977), 445-449.

[15] V. Hutson and J. S. Pym, Applications of functional analysis and operator theory, Aca. Press INC. 1980.

[16] A. J. Klimas, A method for overcoming the velocity space filamentation problem in collisionless plasma model solutions, J. Comput. Phys. 68 (1987), 202-226.

[17] R. J. LeVeque, Numerical methods for conservation laws, Birk. Verlag, 1992.

[18] G. Manfredi, Long time behavior of non linear Landau damping, Phys. Rev. Lett. 79 (1997), 2815-2818.

[19] T. Nakamura and T. Yabe, Cubic interpolated progation scheme for solving the Hyper-Dimensional Vlasov-Poisson equation in phase space, Comput. Phys. Comm. 120 (1999), 122-154.

[20] K. Prarrelmoser, Global classical solutions of the Vlasov-Poisson system in three dimensions for general initial data, J. Differ. Equat. 95 (1992), 281-303.

[21] E.Sonnendrucker, J. Roche, P. Bertrand and A. Ghizzo, The semi-lagrangian method for the numerical resolution of the Vlasov equation, J. Comput. Phys. 149 (1999), 201-220.

[22] E.Sonnendrucker, F. Filbet, A. Friedma, E. Oudet, and J. L. Vay, Vlasov simulations of beams with a moving grid, Comput. Phys. Comm. 164 (2004), 390395.

[23] J. C. Tannehill, D. A. Anderson, and R. H. Pletcher, Computational fluid mechanice and heat transfer, SPT, 1984.

[24] T. Umeda, Vlasov simulation of amplitude-modulated Langmuir waves, Phys. Plasmas 13, 2006.

[25] T. Umeda, A conservative and non-oscillatory scheme for Vlasov code simulations, Earth Plan. Space 60 (2008), 773-779.

[26] S. Ukai and T. Okabe, On classical solutions in the large in time of twodimensional Vlasov's equation, Osaka J. Math. 15 (1978), 245-261.

[27] D. Yi and S. Lee, Fourth-order partial differential equations for image enhancement, AMC 175 (2006), 430-440. 
Department of Mathematics

Air Force Academy

Cheongwon 363-849, Republic of Korea

E-mail: byc8734@hanmail.net

**

Department of Mathematics

Air Force Academy

Cheongwon 363-849, Republic of Korea

E-mail: sbgang@postech.ac.kr

$* * *$

Department of Mathematics

Air Force Academy

Cheongwon 363-849, Republic of Korea

E-mail: kafa04@snu.ac.kr 